\title{
Atom-by-atom structural and chemical analysis by annular dark-field electron microscopy
}

\author{
Ondrej L. Krivanek ${ }^{1}$, Matthew F. Chisholm², Valeria Nicolosi ${ }^{3}$, Timothy J. Pennycook ${ }^{2,4}$, George J. Corbin ${ }^{1}$, \\ Niklas Dellby ${ }^{1}$, Matthew F. Murfittt ${ }^{1}$, Christopher S. Own ${ }^{1}$, Zoltan S. Szilagyi ${ }^{1}$, Mark P. Oxley ${ }^{2,4}$, \\ Sokrates T. Pantelides ${ }^{2,4}$ \& Stephen J. Pennycook ${ }^{2,4}$
}

Direct imaging and chemical identification of all the atoms in a material with unknown three-dimensional structure would constitute a very powerful general analysis tool. Transmission electron microscopy should in principle be able to fulfil this role, as many scientists including Feynman realized early on ${ }^{1}$. It images matter with electrons that scatter strongly from individual atoms and whose wavelengths are about $\mathbf{5 0}$ times smaller than an atom. Recently the technique has advanced greatly owing to the introduction of aberration-corrected optics ${ }^{2-8}$. However, neither electron microscopy nor any other experimental technique has yet been able to resolve and identify all the atoms in a non-periodic material consisting of several atomic species. Here we show that annular dark-field imaging in an aberration-corrected scanning transmission electron microscope optimized for low voltage operation can resolve and identify the chemical type of every atom in monolayer hexagonal boron nitride that contains substitutional defects. Three types of atomic substitutions were found and identified: carbon substituting for boron, carbon substituting for nitrogen, and oxygen substituting for nitrogen. The substitutions caused in-plane distortions in the boron nitride monolayer of about 0.1 A magnitude, which were directly resolved, and verified by density functional theory calculations. The results demonstrate that atom-by-atom structural and chemical analysis of all radiation-damage-resistant atoms present in, and on top of, ultrathin sheets has now become possible.

Solving an atomic structure requires that the locations and the chemical types of all the atoms that make up the structure be determined. In an electron microscope, the atomic locations are determined by imaging the atoms using elastically scattered electrons, and this can be done for both heavy and light atoms ${ }^{2-6,9}$. To determine the chemical species at a similarly high spatial resolution, electron energy-loss spectroscopy (EELS) is normally used ${ }^{7,8,10-13}$. Compared with atom-probe tomography ${ }^{14,15}$, EELS enjoys the advantages that it is non-destructive (at least in principle) and that it collects information on $100 \%$ of the sample's atoms. However, it suffers from limitations that include weak scattering cross-sections, delocalization of the EELS signal, and a lack of spectral features suitable for an identification at high spatial resolution for about $50 \%$ of elements. The EELS technique is able to identify single atoms of different rare earths spaced about a nanometre apart ${ }^{8}$, but unfortunately, it is not able to serve as a general atom-by-atom identification technique in closely packed materials.

In order to identify which atom is which in monolayer boron nitride $(\mathrm{BN})$, bright-field phase-contrast imaging has recently been used $^{16-18}$. However, because the bright-field signal is very similar for boron and nitrogen (see figure $2 \mathrm{~g}$ in ref. 16), the two types of atoms are difficult to distinguish. In a recent study of BN (ref. 18), a through-focus series of many images was processed to extract a phase image of the sample, and this image was averaged over 20 different B-N pair locations, in order to obtain a signal-to-noise ratio that was sufficiently high to distinguish the two types of atoms. The goal of identifying the chemical type of individual atoms within the $\mathrm{BN}$ monolayer, from a single electron micrograph capturing a particular configuration in a changing sample, was therefore not reached.

In the present work, the technique used for atom-by-atom identification at high spatial resolution is annular dark-field (ADF) imaging in a scanning transmission electron microscope (STEM), introduced by Crewe and co-workers and used by them to image single heavy atoms ${ }^{19}$. In ADF STEM, a very small probe of electrons is formed by electron optics consisting of up to about 100 independent elements in today's most advanced electron microscopes, and the probe is rapidly scanned across the sample. The collected dark-field signal is due to Rutherford scattering from the partially screened atomic nucleus, and it increases ${ }^{20}$ with the atomic number $(Z)$ of the atom as about $Z^{1.7}$. This dependence makes the ADF signal from light atoms much weaker than from heavy ones, and the technique has therefore not been used much for imaging light atoms. As we show here, in an aberration-corrected STEM optimized for low-voltage operation, the ADF signal is strong enough to image and identify the chemical type of all atoms, including light ones.

Figure 1a shows a high-magnification ADF STEM image of monolayer $\mathrm{BN}$. The image was recorded at $60 \mathrm{kV}$ primary voltage, which is below the knock-on radiation damage threshold of $78 \mathrm{kV}$ in $\mathrm{BN}$ (ref. 21), and the probe size was about $1.2 \AA$ (see Supplementary Information). The image was rotated to align the principal BN directions with the image edges but not processed in any other way. The BN monolayer was bordered by thicker regions seen on the left side of the image, which included carbon (as was shown by electron energy-loss spectra), heavier atom impurities imaged as isolated bright atoms, and most probably also hydrogen. A lower magnification image showing a larger area of the imaged monolayer and various other sample features including the sample's edge is given in Supplementary Fig. 1.

Individual boron and nitrogen atoms are clearly distinguished by their intensity in Fig. 1. Each single hexagonal ring of the BN structure, for instance the one marked by the green circle in the figure, consists of three brighter nitrogen atoms and three darker boron atoms. The pattern is repeated throughout the image of the monolayer BN. However, there are several deviations from this pattern, such as the hexagonal ring marked by the yellow circle, which shows six atoms with intensities intermediate to those of B and N.

In order to interpret the irregular features correctly, we needed to remove the contribution from the 'tail' of the electron probe to the 

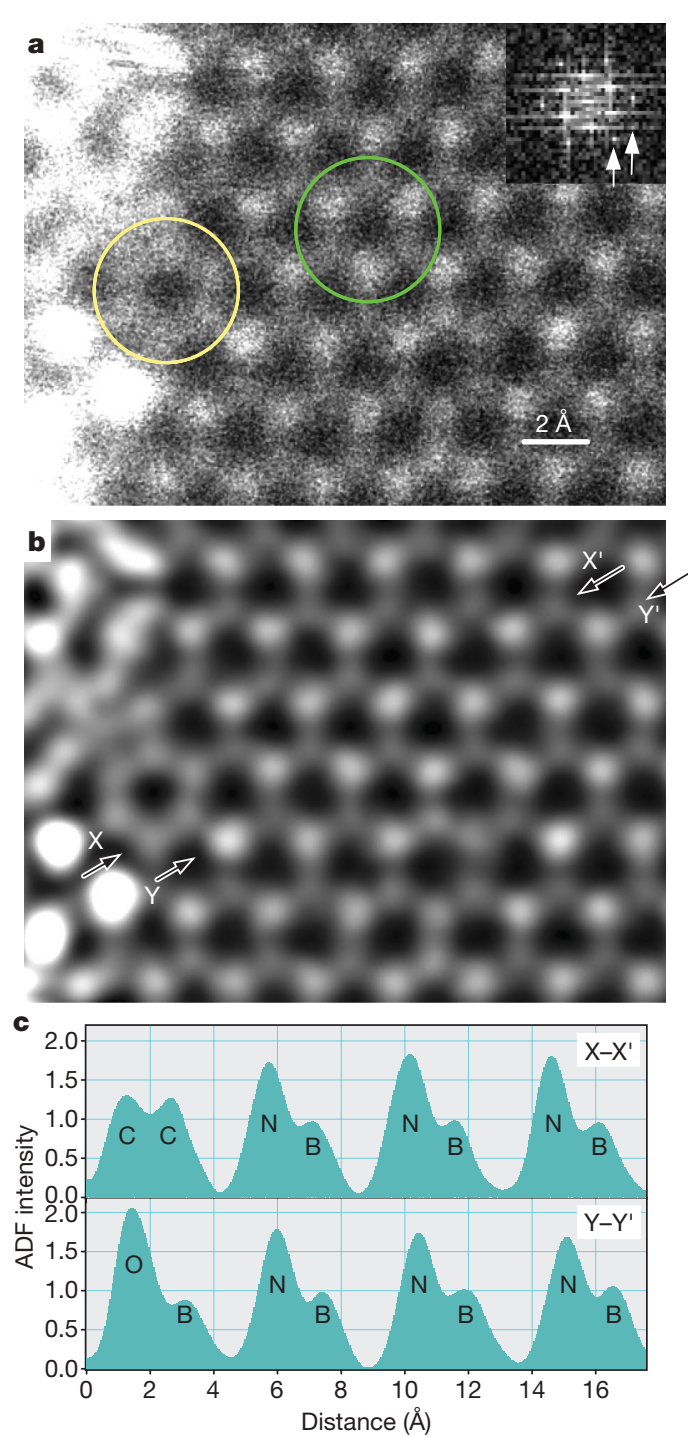

Figure 1 | ADF STEM image of monolayer BN. a, As recorded. b, Corrected for distortion, smoothed, and deconvolved to remove probe tail contributions to nearest neighbours. $c$, Line profiles showing the image intensity (normalized to equal one for a single boron atom) as a function of position in image $\mathbf{b}$ along $\mathrm{X}-\mathrm{X}^{\prime}$ and $\mathrm{Y}-\mathrm{Y}^{\prime}$. The elements giving rise to the peaks seen in the profiles are identified by their chemical symbols. Inset at top right in a shows the Fourier transform of an image area away from the thicker regions. Its two arrows point to $(11 \overline{2} 0)$ and $(20 \overline{2} 0)$ reflections of the hexagonal $\mathrm{BN}$ that correspond to recorded spacings of 1.26 and $1.09 \AA$.

nearest-neighbour sites. If this contribution is present, the tails from the heavier $\mathrm{N}$ atoms contribute more strongly to the $\mathrm{B}$ sites than the tails from the lighter $\mathrm{B}$ atoms contribute to the $\mathrm{N}$ sites, and the intensities at the two sites cannot be compared quantitatively. We deconvolved the image using a procedure (Methods Summary) that removed the tail contribution from the nearest-neighbour sites, and also removed (smoothed) the pixel-to-pixel statistical noise caused by the spreading of the available signal among the many pixels in a massively oversampled image. The deconvolved (and 'un-distorted', see Supplementary Information) version of Fig. 1a is shown in Fig. 1b.

Figure 1c shows line profiles through the two locations marked in Fig. 1b. Profile $\mathrm{X}-\mathrm{X}^{\prime}$ starts with two atoms of the hexagonal ring marked by the yellow circle in Fig. 1a. The ring's atoms are clearly different from the alternating boron and nitrogen atoms shown in the rest of the profile. Carbon $(Z=6)$ substituting for the $\mathrm{B}(Z=5)$ and $\mathrm{N}(Z=7)$ is the likely explanation. Profile $\mathrm{Y}-\mathrm{Y}^{\prime}$ goes through the regular $\mathrm{B}-\mathrm{N}$ pairs, with one exception: the first atom in the profile is significantly brighter, and is probably oxygen $(Z=8)$. Without a
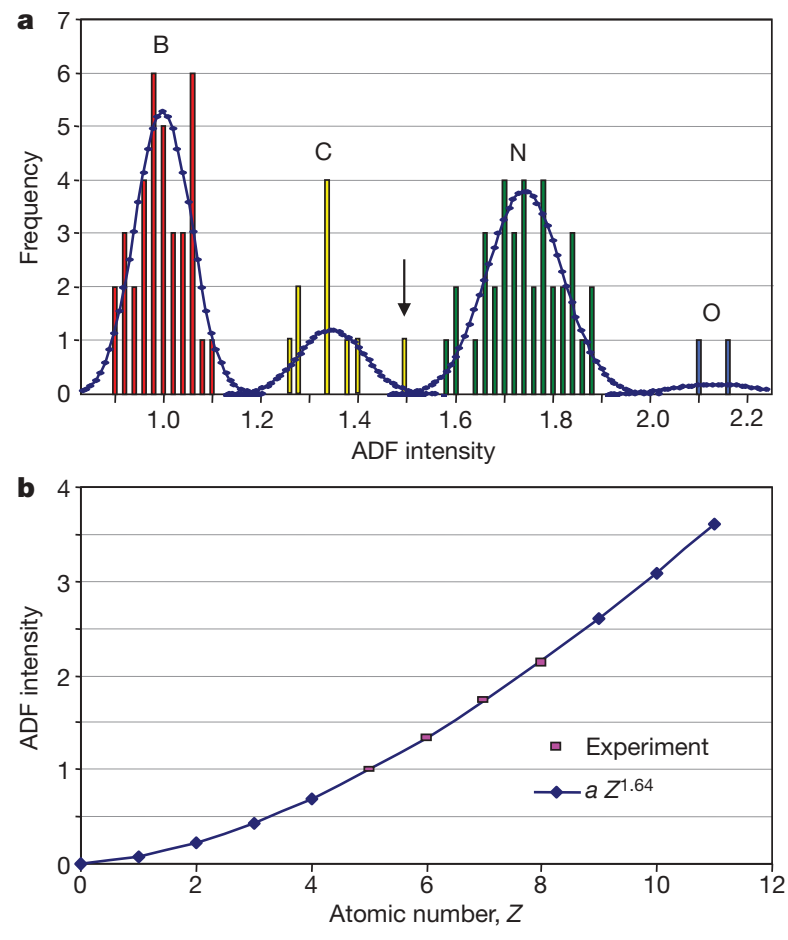

Figure 2 | Analysis of image intensities. a, Histogram of the intensities of atomic image maxima in the monolayer area of Fig. 1b. b. Plot of the average intensities of the different types of atoms versus their atomic number, $Z$. The heights of the rectangles shown for $\mathrm{B}, \mathrm{C}, \mathrm{N}$ and $\mathrm{O}$ correspond to the experimental error in determining the mean of each atomic type's intensity distribution.

quantitative statistical analysis, however, atomic identifications such as these are rather tentative. The appropriate way to quantify the image is to compute a histogram showing the distribution of the atom intensities ${ }^{22,23}$ for all the atoms in a given area, and to use the histogram to determine the probability that the atomic assignments were made correctly. Such an analysis seems not to have been done before on images of atoms of similar $Z$.

Figure $2 \mathrm{a}$ shows a histogram of the peak intensities for all the atoms in the monolayer sample area covered by Fig. $1 \mathrm{~b}$ (that is, excluding atoms in the thicker region on the left side of the figure), normalized such that the centre of the B peak is 1. A theoretical fit for the distributions of the different species of the atoms, based on the standard deviations determined experimentally for the $\mathrm{B}$ and $\mathrm{N}$ atoms, and extrapolated to the $\mathrm{C}$ and $\mathrm{O}$ distributions, is overlaid on the figure. The separation of the histogram peaks is such that every atom in the analysed area could be assigned to a particular species with $>99 \%$ confidence, with one exception: the atom marked by the arrow in the histogram. This atom's intensity was $3 \sigma_{\mathrm{C}}$ from the mean $\mathrm{C}$ value and $5.6 \sigma_{\mathrm{N}}$ from the mean $\mathrm{N}$ value, and it was therefore likely to be carbon at $94 \%$ confidence level. In the examined part of the image, no $\mathrm{N}$ atoms were assigned to B sites or vice versa. This was expected at the $>99.99 \%$ confidence level that applied to not assigning an atomic species differing by $\Delta \mathrm{Z}=2$ from the correct one.

Figure $2 \mathrm{~b}$ shows the dependence of the average intensity of the four types of atoms identified in the sample on their atomic number $Z$, and compares it to a $Z^{1.64}$ model spanning from $Z=1$ to 11 ( $\mathrm{H}$ to $\mathrm{Na}$ ). The match for the observed elements shows no deviation exceeding the statistical noise present in the image. It demonstrates the simple nature of $\mathrm{ADF}$ image contrast, which is able to reveal the chemical identity of non-overlapping atoms very directly. The power of the derived $Z$ dependence is influenced by the deconvolution procedure, and it will also vary slightly depending on the signal collection geometry used and the $Z$ of the studied elements. However, it is expected to remain in the range $1.5-1.8$. 
There were three heavier atoms in the bottom left corner of Fig. 1b, and these were a good match for sodium adatoms sitting on top of $\mathrm{N}$ atoms in a continuation of the $\mathrm{BN}$ monolayer. For much heavier elements, it is useful to consider the expected separation of the histogram peaks, and this is done in the Supplementary Information. The analysis shows that with an electron dose of about $2 \times 10^{7}$ electrons per $\AA^{2}$, non-overlapping stable atoms of all elements should be identifiable unambiguously by their ADF intensities.

In samples other than monolayers, the atoms typically lie on a background of other atoms. In the case of unknown structures resting on top of thin $\mathrm{BN}$ and graphene, the underlying substrate can be subtracted with no trace remaining other than the statistical noise of the substrate image, and simple molecules and other structures should be analysable using a methodology similar to that used here. For structures with more complicated projections, ADF threedimensional tomography ${ }^{24}$ carried out at a resolution similar to that attained here will be able to derive the correct sample structure even when the individual projections are quite complicated. The major practical limit will then come from the sample changing from image to image due to thermal motion and radiation damage, but this may be manageable if the sample is cooled and the operating voltage is lowered further.

Figure 3 shows a diagram of the atomic structure that corresponds to the impurity atoms identified in the monolayer, superimposed on the deconvolved experimental image. Carbon atoms are only seen to substitute for B-N pairs, not for individual boron or nitrogen atoms. Oxygen, on the other hand, substitutes for single $\mathrm{N}$ atoms. The atomic positions in the diagram were determined by a density functional theory (DFT) relaxation (see Supplementary Information), whose input was a single $\mathrm{BN}$ layer containing the substitutional defects identified by the histogram analysis.

Close inspection of Figs $1 \mathrm{~b}$ and 3 shows that there were significant distortions in the lattice next to the two oxygen atoms, whose nearest neighbours were significantly further away than the B-N distance of $1.45 \AA$. One very visible result of these distortions was that in the hexagonal ring of $\mathrm{C}$ atoms, the $\mathrm{C}$ nearest neighbour of the adjacent $\mathrm{O}$ atom appears 'pushed' into the carbon ring. This is seen both in the experimental image, which shows the average distance between the two oxygen atoms and their nearest neighbours (averaged over the six nearest neighbours of the two atoms) to be stretched by $0.14 \pm 0.08 \AA$ relative to the $\mathrm{B}-\mathrm{N}$ distance of $1.45 \AA$, and in the DFT-relaxed model, which shows an $\mathrm{O}-\mathrm{C}$ stretch of $0.09 \AA$. This demonstrates that even in the presence of statistical noise that shifts the individual atomic images by small random amounts, ADF imaging is at present able to track atomic displacements with a precision of about $0.1 \AA$.

Mixtures of boron, carbon and nitrogen can be synthesized into hexagonal sheets and nanotubes ${ }^{25,26}$. Our starting $\mathrm{BN}$ material

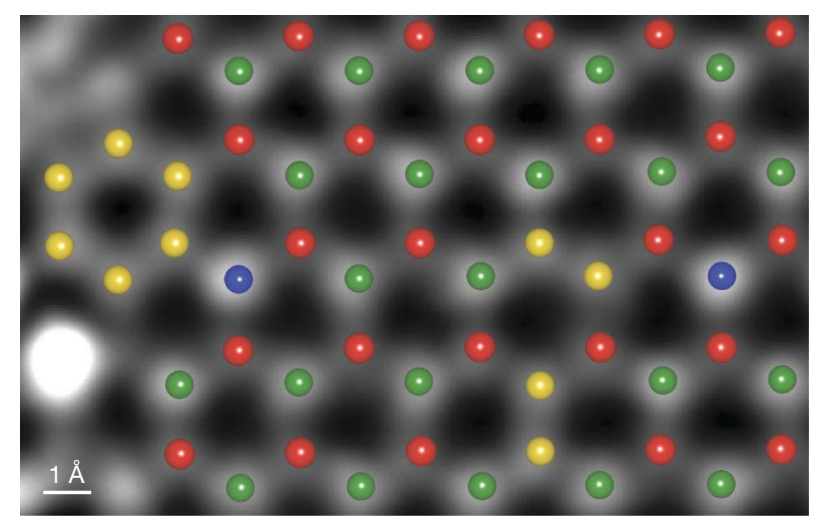

Figure 3 | The atomic structure determined by the histogram analysis. Part of a DFT simulation of a single BN layer containing the experimentally observed substitutional impurities overlaid on the corresponding part of the experimental image. Red, B; yellow, C; green, $\mathrm{N}$; blue, $\mathrm{O}$. contained enough impurities to account for the substitutional atoms (see Supplementary Information). However, a three-atom hole was observed in the single BN layer just 2 min before the image of Fig. 1 was taken (see Supplementary Information), precisely where the hexagonal $\mathrm{C}$ ring is in Fig. 1a. Another hole was observed even earlier, roughly where the right-side $\mathrm{O}$ atom and the four $\mathrm{C}$ impurities separate from the $\mathrm{C}$ ring were imaged in Fig. 1. An image of the same area recorded earlier still showed no holes and no substitutions. This demonstrates that the substitutions arose when holes opened up in the material due to the bombardment by the electron beam, and were subsequently filled with $\mathrm{C}$ and $\mathrm{O}$ atoms available in the overlayers. Drilling holes with an atomic-size electron beam and filling them with atoms available in nearby reservoirs may be a promising method for constructing custom-designed structures with atomic precision.

In summary, ADF imaging in an aberration-corrected STEM operated at $60 \mathrm{kV}$ primary voltage has allowed us to determine the positions and the chemical types of all the atoms in a significant part of an unknown, non-periodic material. Looking towards the future, we note that the ADF signal originates from electron scattering by the atomic nucleus, and that the potentially attainable ADF resolution is therefore much sharper than the 'conventional' atomic size that is determined by the diameter of the outermost electron orbitals. As instrumentation advances improve the resolution of electron microscopes even further in the future, the ADF signal from individual atoms will be focused into smaller and smaller image areas. This will improve the signal-to-noise ratios of individual atomic images recorded at a given electron dose, and the atoms will become easier to identify unambiguously even in complicated projections. Atomby-atom structural and chemical analysis will then become available for a wide range of materials, precisely as envisaged by Feynman ${ }^{1}$.

\section{METHODS SUMMARY}

The samples were prepared by liquid phase exfoliation ${ }^{27}$ of bulk hexagonal BN in $\mathrm{N}$-methyl-2-pyrrolidone. This gave thin flakes of BN that included monolayer areas, and the flakes were suspended on holey carbon films. Monolayer areas situated over holes in the support film were imaged in a Nion UltraSTEM scanning transmission electron microscope using a cold field emission electron source and a corrector of third and fifth order aberrations ${ }^{28}$, with a probe current of $\sim 50 \mathrm{pA}$ and a typical dose of $6 \times 10^{6}$ electrons per $\AA^{2}$ per high resolution image. The angular range of the collected electrons was about 58-200 mrad halfangle. As documented more extensively in Supplementary Information, the kernel used to deconvolve the experimental image consisted of two rotationally symmetric Gaussians: a positive Gaussian that smoothed the pixel-to-pixel intensity variation, and a negative Gaussian that added a negative 'skirt' to the image of each atom so as to null the extended probe tail at the location of the nearest neighbours. The skirt extended from $0.9 \AA$ to $1.8 \AA$ from the centre of the atom's image, and its peak intensity was $-10 \%$ of the image maximum.

Received 3 November 2009; accepted 29 January 2010.

1. Feynman, R. P. in Feynman and Computation (ed. Hey, J. G. ) 63-76 (Perseus Press, Cambridge, Massachusetts, 1999); text of original 1959 lecture also available at 〈http://feynman.caltech.edu/plenty.html〉 (2001).

2. Haider, M. et al. Electron microscopy image enhanced. Nature 392, 768-769 (1998).

3. Batson, P. E., Dellby, N. \& Krivanek, O. L. Sub-ångstrom resolution using aberration corrected electron optics. Nature 418, 617-620 (2002).

4. Hawkes, P. W. (ed.) Aberration-Corrected Electron Microscopy (Advances in Imaging and Electron Physics, Vol. 153, Elsevier, 2008).

5. Girit, C. Ö. et al. Graphene at the edge: stability and dynamics. Science 323 1705-1708 (2009)

6. Jia, C. L., Lentzen, M. \& Urban, K. Atomic resolution imaging of oxygen in perovskite ceramics. Science 299, 870-873 (2003).

7. Muller, D. A. et al. Atomic-scale chemical imaging of composition and bonding by aberration-corrected microscopy. Science 319, 1073-1076 (2008).

8. Suenaga, K. et al. Visualizing and identifying single atoms using electron energyloss spectroscopy with low accelerating voltage. Nature Chem. 1, 415-418 (2009).

9. Kisielowski, C. et al. Imaging columns of the light elements carbon, nitrogen and oxygen with sub Ångstrom resolution. Ultramicroscopy 89, 243-263 (2001).

10. Suenaga, K. et al. Element-selective single atom imaging. Science 290, 2280-2282 (2000).

11. Leapman, R. D. Detecting single atoms of calcium and iron in biological structures by electron energy-loss spectrum-imaging. J. Microsc. 210, 5-15 (2003). 
12. Varela, M. et al. Spectroscopic imaging of single atoms within a bulk solid. Phys. Rev. Lett. 92, 095502 (2004).

13. Egerton, R. F. Electron Energy Loss Spectroscopy in the Electron Microscope 2nd edn (Plenum, 1996)

14. Miller, M. K. Atom Probe Tomography: Analysis at the Atomic Level (Kluwer Academic/Plenum, 2000)

15. Kelly, T. F. \& Miller, M. K. Atom probe tomography. Rev. Sci. Instrum. 78, 031101 (2007)

16. Meyer, J. C., Chuvilin, A., Algara-Siller, G., Biskupek, J. \& Kaiser, U. Selective sputtering and atomic resolution imaging of atomically thin boron nitride membranes. Nano Lett. 9, 2683-2689 (2009).

17. Jin, C., Lin, F., Suenaga, K. \& lijima, S. Fabrication of a freestanding boron nitride single layer and its defect assignments. Phys. Rev. Lett. 102, 195505 (2009).

18. Alem, N. et al. Atomically thin hexagonal boron nitride probed by ultrahighresolution transmission electron microscopy. Phys. Rev. B 80, 155425 (2009).

19. Crewe, A. V., Wall, J. \& Langmore, J. Visibility of single atoms. Science 168, 1338-1340 (1970)

20. Hartel, P., Rose, H. \& Dignes, C. Conditions and reasons for incoherent imaging in STEM. Ultramicroscopy 63, 93-114 (1996).

21. Zobelli, A., Gloter, A., Ewels, C. P., Seifert, G. \& Colliex, C. Electron knock-on cross section of carbon and boron nitride nanotubes. Phys. Rev. B 75, 245402 (2007).

22. Issacson, M., Kopf, D., Ohtsuki, M. \& Utlaut, M. Atomic imaging using the dark field annular detector in the STEM. Ultramicroscopy 4, 101-104 (1979).

23. Voyles, P. M., Muller, D. A., Grazul, J. L., Citrin, P. H. \& Gossman, H.-J. L. Atomicscale imaging of individual dopant atoms and clusters in highly $\mathrm{n}$-type bulk Si. Nature 416, 826-829 (2002).

24. Midgley, P. A. \& Weyland, M. 3D electron microscopy in the physical sciences: the development of Z-contrast and EFTEM tomography. Ultramicroscopy 96, 413-431 (2003).

25. Stephan, O. et al. Doping graphitic and carbon nanotube structures with boron and nitrogen. Science 266, 1683-1685 (1994).
26. Kawaguchi, M., Kuroda, S. \& Muramatsu, Y. Electronic structure and intercalation chemistry of graphite-like layered material with a composition of $\mathrm{BCB}_{6} \mathrm{BN}$. J. Phys. Chem. Solids 69, 1171-1178 (2008).

27. Hernandez, Y. et al. High yield production of graphene by liquid phase exfoliation of graphite. Nature Nanotechnol. 3, 563-578 (2008).

28. Krivanek, O. L. et al. An electron microscope for the aberration-corrected era. Ultramicroscopy 108, 179-195 (2008).

Supplementary Information is linked to the online version of the paper at www.nature.com/nature.

Acknowledgements We thank L. M. Brown, J. N. Coleman, P. Rez and J. C. H. Spence for discussions. Research at Oak Ridge National Laboratory (M.F.C., T.J.P., M.P.O., S.T.P. and S.J.P.) was sponsored by the Division of Materials Sciences and Engineering of the US Department of Energy. Research at Vanderbilt was supported in part by the US Department of Energy grant DE-FG02-09ER46554 and the McMinn Endowment. Computations were performed at the National Energy Research Scientific Computing Center at Lawrence Berkeley National Laboratory.

Author Contributions O.L.K. initiated the project and wrote the paper, M.F.C., N.D. O.L.K. and M.F.M. recorded preliminary experimental data, M.F.C. improved the imaging and recorded the data used in the paper, V.N. prepared the samples, O.L.K. and N.D. performed data processing and analysis, T.J.P. and S.T.P. performed DFT calculations, M.P.O. performed image calculations, S.J.P. initiated the aberration-corrected microscopy project at ORNL and advised on the paper, and G.J.C., N.D., O.L.K., M.F.M, C.S.O. and Z.S.S. designed and built the electron microscope. All the authors read and commented on the manuscript.

Author Information Reprints and permissions information is available at www.nature.com/reprints. The authors declare competing financial interests: details accompany the full-text HTML version of the paper at www.nature.com/ nature. Correspondence and requests for materials should be addressed to O.L.K. (krivanek@nion.com). 